\title{
Sekiguchi-Debiard operators at infinity
}

\author{
M. L. Nazarov and E. K. Sklyanin \\ Department of Mathematics, University of York, York YO10 5DD, United Kingdom
}

\begin{abstract}
We construct a family of pairwise commuting operators such that the Jack symmetric functions of infinitely many variables $x_{1}, x_{2}, \ldots$ are their eigenfunctions. These operators are defined as limits at $N \rightarrow \infty$ of renormalised Sekiguchi-Debiard operators acting on symmetric polynomials in the variables $x_{1}, \ldots, x_{N}$. They are differential operators in terms of the power sum variables $p_{n}=x_{1}^{n}+x_{2}^{n}+\ldots$ and we compute their symbols by using the Jack reproducing kernel. Our result yields a hierarchy of commuting Hamiltonians for the quantum Calogero-Sutherland model with infinite number of bosonic particles in terms of the collective variables of the model. Our result also yields the elementary step operators for the Jack symmetric functions.
\end{abstract}




\section{Introduction}

1.1. Calogero-Sutherland model. This quantum model describes a system of $N$ bosonic particles on a circle $\mathbb{R} / \pi \mathbb{Z}$ with the Hamiltonian $[5,19,20]$

$$
H_{\mathrm{CS}}=-\frac{1}{2} \sum_{i} \frac{\partial^{2}}{\partial q_{i}^{2}}+\sum_{i<j} \frac{\beta(\beta-1)}{\sin ^{2}\left(q_{i}-q_{j}\right)}
$$

where $0 \leqslant q_{1}, \ldots, q_{N}<\pi$. Being translationally invariant $H_{\mathrm{CS}}$ commutes with the momentum operator

$$
P_{\mathrm{CS}}=-\mathrm{i} \sum_{j} \frac{\partial}{\partial q_{j}} .
$$

After eliminating the vacuum factor

$$
\omega=\left|\prod_{i<j} \sin \left(q_{i}-q_{j}\right)\right|^{\beta}
$$

and then passing to the exponential variables $x_{j}=\exp \left(2 \mathrm{i} q_{j}\right)$ and the parameter $\alpha=\beta^{-1}$ more common in the mathematical literature, the Hamiltonian becomes

$$
\omega^{-1} \circ H_{\mathrm{CS}} \circ \omega=\frac{2}{\alpha} H_{N}^{(2)}+\frac{N^{3}-N}{6 \alpha^{2}}
$$

where

$$
H_{N}^{(2)}=\alpha \sum_{i}\left(x_{i} \frac{\partial}{\partial x_{i}}\right)^{2}+\sum_{i<j} \frac{x_{i}+x_{j}}{x_{i}-x_{j}}\left(x_{i} \frac{\partial}{\partial x_{i}}-x_{j} \frac{\partial}{\partial x_{j}}\right) .
$$

Respectively, $P_{\mathrm{CS}}$ gives rise to the operator

$$
H_{N}^{(1)}=\frac{1}{2} P_{\mathrm{CS}}=\sum_{j} x_{j} \frac{\partial}{\partial x_{j}} .
$$

The operators $H_{N}^{(1)}$ and $H_{N}^{(2)}$ commute and act on symmetric polynomials of the variables $x_{1}, \ldots, x_{N}$. It is known that both operators can be included into a quantum integrable hierarchy, that is into a polynomial ring of commuting differential operators with $N$ generators of orders $1, \ldots, N$ respectively, called the Sekiguchi-Debiard operators $[6,16]$. The Jack symmetric polynomials [8] are joint eigenfunctions of the hierarchy. They are labelled by partitions of $0,1,2, \ldots$. with no more than $N$ non-zero parts.

In combinatorics it is quite common to extend various symmetric polynomials to an infinite countable set of variables. These extensions are called symmetric functions. In particular, the extensions of the Jack symmetric polynomials are well studied [8]. They are labelled by partitions of $0,1,2, \ldots$ However, no explicit expressions for higher commuting Hamiltonians corresponding to the infinite set of variables have been yet available in the Jack case, with an exception of a few lower order operators. The main purpose of the present article is to fill up the gap, by studying the limits of the Sekiguchi-Debiard operators as $N \rightarrow \infty$ and giving explicit expressions for the resulting commuting Hamiltonians. 
As another application of our result, we construct elementary step operators for the Jack symmetric functions. In terms of the labels, our operators correspond to decreasing by 1 any given non-zero part of a partion, and to the operation on partitions inverse to that, see our formulas (2.31) and (2.29) respectively. For the origins of this construction see the work [18] and references therein. For related but different results on the Jack polynomials see the work [7].

1.2. Collective variables. The standard way to treat the $N=\infty$ case is to rewrite the Hamiltonian (1.1) in terms of the power sums (the "collective variables" in the condensed matter physics terminology)

$$
x_{1}^{n}+\ldots+x_{N}^{n} \quad \text { where } \quad n=1, \ldots, N
$$

and to take the limit at $N \rightarrow \infty$ afterwards. Denoting the limit of the power sum (1.2) by $p_{n}$ where $n=1,2, \ldots$ the resulting Hamiltonian reads [9]

$$
\begin{gathered}
H^{(2)}=\lim _{N \rightarrow \infty} \alpha\left(H_{N}^{(2)}-N H_{N}^{(1)}\right)= \\
\sum_{m, n=1}^{\infty}\left(\alpha(m+n) p_{m} p_{n} \frac{\partial}{\partial p_{m+n}}+\alpha^{2} m n p_{m+n} \frac{\partial^{2}}{\partial p_{m} \partial p_{n}}\right)+ \\
(\alpha-1) \sum_{n=1}^{\infty} \alpha n^{2} p_{n} \frac{\partial}{\partial p_{n}} .
\end{gathered}
$$

The first and the second summands in the middle line of the above display are known as splitting terms and joining terms respectively, see also [3] and [4].

Consider the vector space $\Lambda=\mathbb{C}\left[p_{1}, p_{2}, \ldots\right]$ and equip it with the operators $a_{n}$ where $n \in \mathbb{Z} \backslash\{0\}$, defined on the polynomials $f \in \Lambda$ by

$$
a_{n}(f)=\left\{\begin{array}{cc}
p_{-n} f & \text { if } \quad n<0 \\
\alpha n \partial f / \partial p_{n} & \text { if } \quad n>0
\end{array}\right.
$$

see also the equality $(2.14)$ below. The operators $a_{n}$ satisfy the relations

$$
\left[a_{m}, a_{n}\right]=m \alpha \delta_{m+n, 0} .
$$

Thus $\Lambda$ becomes a highest weight module for an infinite-dimensional Heisenberg Lie algebra. In terms of the operators $a_{n}$ the Hamiltonian (1.3) takes the form

$$
H^{(2)}=\sum_{m, n=1}^{\infty}\left(a_{-m} a_{-n} a_{m+n}+a_{-m-n} a_{m} a_{n}\right)+(\alpha-1) \sum_{n=1}^{\infty} n a_{-n} a_{n} .
$$

Similarly, the $H_{N}^{(1)}$ yields a first order differential operator commuting with $H^{(2)}$

$$
H^{(1)}=\lim _{N \rightarrow \infty} \alpha H_{N}^{(1)}=\sum_{n=1}^{\infty} \alpha n p_{n} \frac{\partial}{\partial p_{n}}=\sum_{n=1}^{\infty} a_{-n} a_{n} .
$$


1.3. Quantum field theory. In terms of the field $\varphi(s)$ on the circle $S^{1} \equiv \mathbb{R} / 2 \pi \mathbb{Z}$

$$
\varphi(s)=\sum_{n \neq 0} a_{n} \exp (-\mathrm{i} n s) \quad \text { for } \quad s \in S^{1}
$$

the commutation relations (1.4) read

$$
[\varphi(s), \varphi(t)]=2 \pi \mathrm{i} \alpha \delta^{\prime}(s-t)
$$

Setting $a_{0}=0$ and using the Wick normal ordering : : with the operators $a_{n}$ for $n<0$ to the left and for $n>0$ to the right, the Hamiltonian $H^{(1)}$ becomes

$$
\frac{1}{2 \alpha} \sum_{m+n=0}: a_{m} a_{n}:=\frac{1}{2 \alpha} \int_{S^{1}} \frac{\mathrm{d} s}{2 \pi}: \varphi^{2}(s):
$$

while $H^{(2)}$ becomes the Hamiltonian of quantized Benjamin-Ono equation $[1,13]$

$$
\begin{gathered}
\frac{1}{3} \sum_{l+m+n=0}: a_{l} a_{m} a_{n}:+\frac{\alpha-1}{2} \sum_{m+n=0}|n|: a_{m} a_{n}:= \\
\int_{S^{1}} \frac{\mathrm{d} s}{2 \pi}\left(\frac{1}{3}: \varphi^{3}(s):+\frac{1-\alpha}{2}: \varphi^{\prime}(s)(\mathcal{H} \varphi)(s):\right)
\end{gathered}
$$

where $\mathcal{H}$ stands for the Hilbert transform

$$
(\mathcal{H} \varphi)(s)=\text { p.v. } \int_{S^{1}} \frac{\mathrm{d} t}{2 \pi} \cot \frac{s-t}{2} \varphi(t)
$$

In the particular case $\alpha=1$ the Jack symmetric polynomials degenerate into Schur polynomials. The Benjamin-Ono equation respectively degenerates into the dispersionless KdV (also called Burgers) equation. An explicit construction of a countable set of commuting Hamiltonians for the quantum dispersionless $\mathrm{KdV}$ can be obtained via boson-fermion correspondence and is available in terms of recurrence relations [12] or a generating function [11,14].

The higher quantum Hamiltonians for any parameter $\alpha$ are constructed in the present article, see the theorem in Subsection 2.5. Note that in the case $\alpha=1$ our Hamiltonians $A^{(k)}$ are different from those considered in $[11,12,14]$ being rather their polynomial combinations, see for example (2.16) and (2.17) below.

In their turn, Jack symmetric polynomials can be regarded as degenerations of the Macdonald polynomials in the variables $x_{1}, \ldots, x_{N}$ also depending on two formal parameters $q$ and $t$. The Jack case corresponds to $q=t^{\alpha}$ where $t \rightarrow 1$. The Sekiguchi-Debiard operators can be then regarded as degenerations of the Macdonald operators [8] acting on the symmetric polynomials in $x_{1}, \ldots, x_{N}$. Our theorem generalizes to the Macdonald case [10], see also the earlier works $[2,17]$. 
1.4. Plan of the article. In the next section we recall some basic facts from the theory of symmetric functions and set up the notation. We then introduce the Jack polynomials and Sekiguchi-Debiard differential operators. Our main tool is the notion of the symbol of an operator relative to the reproducing kernel for Jack polynomials. After establishing the basics, we state our main result which is an explicit formula for the symbol of the generating function of commuting Hamiltonians. Then we explicitly construct our elementary step operators for the Jack symmetric functions. We finish Section 2 with reducing the proof of our theorem to certain determinantal identities which are then proved in Section 3.

In this article we generally keep to the notation of the book [8] for symmetric functions. When using the results from [8] we will simply indicate their numbers within the book. For example, the statement (1.11) from Chapter I of the book will be referred to as [I.1.11] assuming it is from [8]. We do not number our own lemmas, propositions, theorems or corollaries because we have only one of each.

\section{Symmetric functions}

2.1. Monomial functions. Fix any field $\mathbb{F}$. For any positive integer $N$ denote by $\Lambda_{N}$ the $\mathbb{F}$-algebra of symmetric polynomials in $N$ variables $x_{1}, \ldots, x_{N}$. The algebra $\Lambda_{N}$ is graded by the polynomial degree. The substitution $x_{N}=0$ defines a homomorphism $\Lambda_{N} \rightarrow \Lambda_{N-1}$ preserving the degree. Here $\Lambda_{0}=\mathbb{F}$. The inverse limit of the sequence

$$
\Lambda_{1} \leftarrow \Lambda_{2} \leftarrow \ldots
$$

in the category of graded algebras is denoted by $\Lambda$. The elements of $\Lambda$ are called symmetric functions. Following [8] we will introduce some standard bases of $\Lambda$.

Let $\lambda=\left(\lambda_{1}, \lambda_{2}, \ldots\right)$ be any partition of $0,1,2, \ldots$ The number of non-zero parts is called the length of $\lambda$ and is denoted by $\ell(\lambda)$. If $\ell(\lambda) \leqslant N$ then the sum of all distinct monomials obtained by permuting the $N$ variables in $x_{1}^{\lambda_{1}} \ldots x_{N}^{\lambda_{N}}$ is denoted by $m_{\lambda}\left(x_{1}, \ldots, x_{N}\right)$. The symmetric polynomials $m_{\lambda}\left(x_{1}, \ldots, x_{N}\right)$ with $\ell(\lambda) \leqslant N$ form a basis of the vector space $\Lambda_{N}$. By definition, for $\ell(\lambda) \leqslant N$

$$
m_{\lambda}\left(x_{1}, \ldots, x_{N}\right)=\sum_{1 \leqslant i_{1}<\ldots<i_{k} \leqslant N} \sum_{\sigma \in \mathfrak{S}_{k}} d_{\lambda}^{-1} x_{i_{\sigma(1)}}^{\lambda_{1}} \ldots x_{i_{\sigma(k)}}^{\lambda_{k}}
$$

where we write $k$ instead of $\ell(\lambda)$. Here $\mathfrak{S}_{k}$ is the symmetric group permuting the numbers $1, \ldots, k$ and

$$
d_{\lambda}=k_{1} ! k_{2} ! \ldots
$$

if $k_{1}, k_{2}, \ldots$ are the respective multiplicites of the parts $1,2, \ldots$ of $\lambda$. Further,

$$
m_{\lambda}\left(x_{1}, \ldots, x_{N-1}, 0\right)=\left\{\begin{array}{ccc}
m_{\lambda}\left(x_{1}, \ldots, x_{N-1}\right) & \text { if } & \ell(\lambda)<N \\
0 & \text { if } & \ell(\lambda)=N .
\end{array}\right.
$$

Hence for any fixed partition $\lambda$ the sequence of polynomials $m_{\lambda}\left(x_{1}, \ldots, x_{N}\right)$ with $N \geqslant \ell(\lambda)$ has a limit in $\Lambda$. This limit is called the monomial symmetric function corresponding to $\lambda$. Simply omitting the variables, we will denote the limit by $m_{\lambda}$. With $\lambda$ ranging over all partitions of $0,1,2 \ldots$ the symmetric functions $m_{\lambda}$ form a basis of the vector space $\Lambda$. Note that if $\ell(\lambda)=0$ then we set $m_{\lambda}=1$. 
2.2. Power sums. For each $n=1,2, \ldots$ denote $p_{n}\left(x_{1}, \ldots, x_{N}\right)=x_{1}^{n}+\ldots+x_{N}^{n}$. When the index $n$ is fixed the sequence of symmetric polynomials $p_{n}\left(x_{1}, \ldots, x_{N}\right)$ with $N=1,2, \ldots$ has a limit in $\Lambda$, called the power sum symmetric function of degree $n$. We denote the limit by $p_{n}$. More generally, for any partition $\lambda$ put

$$
p_{\lambda}=p_{\lambda_{1}} p_{\lambda_{2}} \ldots
$$

where we set $p_{0}=1$. The elements $p_{\lambda}$ form another basis of $\Lambda$. In other words, the elements $p_{1}, p_{2}, \ldots$ are free generators of the commutative algebra $\Lambda$ over $\mathbb{F}$.

The basis of $p_{\lambda}$ can be related to the basis of monomial symmetric functions as follows. For any two partitions $\lambda$ and $\mu$ denote by $R_{\lambda \mu}$ the number of mappings $\theta:\{1, \ldots, \ell(\mu)\} \rightarrow\{1,2, \ldots\}$ such that

$$
\sum_{\theta(j)=i} \mu_{j}=\lambda_{i} \quad \text { for each } i=1,2, \ldots
$$

For any such $\theta$ the partition $\mu$ in (2.5) is called a refinement of $\lambda$. Note that if $R_{\lambda \mu} \neq 0$ then $\lambda$ and $\mu$ are partitions of the same number. Moreover, then by [I.6.10] we have $\mu \leqslant \lambda$ in the natural partial ordering of partitions:

$$
\mu_{1} \leqslant \lambda_{1}, \mu_{1}+\mu_{2} \leqslant \lambda_{1}+\lambda_{2}, \ldots .
$$

By [I.6.9] we have

$$
p_{\mu}=\sum_{\mu} R_{\lambda \mu} m_{\lambda}
$$

2.3. Jack functions. Now let $\mathbb{F}$ be the field $\mathbb{Q}(\alpha)$ where $\alpha$ is another variable. Define a bilinear form $\langle$,$\rangle on the vector space \Lambda$ by setting for any $\lambda$ and $\mu$

$$
\left\langle p_{\lambda}, p_{\mu}\right\rangle=\alpha^{\ell(\lambda)} z_{\lambda} \delta_{\lambda \mu}
$$

where

$$
z_{\lambda}=1^{k_{1}} k_{1} ! 2^{k_{2}} k_{2} ! \ldots
$$

in the notation (2.2). This form is obviously symmetric and non-degenerate. By [Ex. VI.4.2] there exists a unique family of elements $P_{\lambda} \in \Lambda$ such that

$$
\left\langle P_{\lambda}, P_{\mu}\right\rangle=0 \quad \text { for } \quad \lambda \neq \mu
$$

and such that any $P_{\lambda}$ equals $m_{\lambda}$ plus a linear combination of the elements $m_{\mu}$ with $\mu<\lambda$ in the natural partial ordering. The elements $P_{\lambda} \in \Lambda$ are called the Jack symmetric functions. Alternatively, they can be defined as follows.

Denote by $\Delta\left(x_{1}, \ldots, x_{N}\right)$ the Vandermonde polynomial of $N$ variables

$$
\operatorname{det}\left[x_{i}^{N-j}\right]_{i, j=1}^{N}=\prod_{1 \leqslant i<j \leqslant N}\left(x_{i}-x_{j}\right) .
$$

Put

$$
S_{N}(u)=\Delta\left(x_{1}, \ldots, x_{N}\right)^{-1} \cdot \operatorname{det}\left[x_{i}^{N-j}\left(u+j-1-\alpha x_{i} \partial_{i}\right)\right]_{i, j=1}^{N}
$$


where $u$ is a variable and $\partial_{i}$ is the operator of partial derivation relative to $x_{i}$. Here the determinant is defined as the alternated sum

$$
\sum_{\sigma \in \mathfrak{S}_{N}}(-1)^{\sigma} \prod_{i=1}^{N}\left(x_{i}^{N-\sigma(i)}\left(u+\sigma(i)-1-\alpha x_{i} \partial_{i}\right)\right)
$$

where as usual $(-1)^{\sigma}$ denotes the sign of permutation $\sigma$. In every product over $i=1, \ldots, N$ appearing in (2.9) the operator factors pairwise commute, hence their ordering does not matter. Further, $S_{N}(u)$ is a polynomial in the variable $u$ with pairwise commuting operator coefficients preserving the space $\Lambda_{N}$, see for instance [Ex. VI.3.1]. We will call the restrictions of these coefficients to the space $\Lambda_{N}$ the Sekiguchi-Debiard operators. By [Ex. VI.4.2] the latter operators have a common eigenbasis in $\Lambda_{N}$ parametrized by partitions $\lambda$ of length $\ell(\lambda) \leqslant N$. The eigenvectors are called the Jack symmetric polynomials.

For each $\lambda$ with $\ell(\lambda) \leqslant N$ there is an eigenvector denoted by $P_{\lambda}\left(x_{1}, \ldots, x_{N}\right)$ which is equal to $m_{\lambda}\left(x_{1}, \ldots, x_{N}\right)$ plus a linear combination of the polynomials $m_{\mu}\left(x_{1}, \ldots, x_{N}\right)$ with $\mu<\lambda$ and $\ell(\mu) \leqslant N$. It turns out that each coefficient in this linear combination does not depend on $N$. Note that if $\lambda$ and $\mu$ are any two partitions of the same number such that $\lambda \geqslant \mu$, then $\ell(\lambda) \leqslant \ell(\mu)$ due to [I.1.11]. It follows that the polynomials $P_{\lambda}\left(x_{1}, \ldots, x_{N}\right)$ enjoy the same stability property as the polynomials $m_{\lambda}\left(x_{1}, \ldots, x_{N}\right)$ in $(2.3)$ :

$$
P_{\lambda}\left(x_{1}, \ldots, x_{N-1}, 0\right)=\left\{\begin{array}{ccc}
P_{\lambda}\left(x_{1}, \ldots, x_{N-1}\right) & \text { if } & \ell(\lambda)<N \\
0 & \text { if } & \ell(\lambda)=N
\end{array}\right.
$$

In particular, the sequence of polynomials $P_{\lambda}\left(x_{1}, \ldots, x_{N}\right)$ with $N \geqslant \ell(\lambda)$ has a limit in $\Lambda$. This is exactly the Jack symmetric function $P_{\lambda}$. The eigenvalues of Sekiguchi-Debiard operators acting on $\Lambda_{N}$ are also known. By [Ex. VI.4.2]

$$
S_{N}(u) P_{\lambda}\left(x_{1}, \ldots, x_{N}\right)=\prod_{i=1}^{N}\left(u+i-1-\alpha \lambda_{i}\right) \cdot P_{\lambda}\left(x_{1}, \ldots, x_{N}\right) .
$$

2.4. Reproducing kernel. In this subsection we will regard the elements of $\Lambda$ as infinite sums of finite products of the variables $x_{1}, x_{2}, \ldots$. For instance, we have

$$
p_{n}=x_{1}^{n}+x_{2}^{n}+\ldots
$$

for any $n \geqslant 1$. When we need to distinguish $x_{1}, x_{2}, \ldots$ from any other variables, we will write $f\left(x_{1}, x_{2}, \ldots\right)$ instead of any $f \in \Lambda$. Now let $y_{1}, y_{2}, \ldots$ be variables independent of $x_{1}, x_{2}, \ldots$. According to [VI.10.4] with the bilinear form (2.7) one associates the reproducing kernel

$$
\Pi=\prod_{i, j=1}^{\infty}\left(1-x_{i} y_{j}\right)^{-1 / \alpha}
$$

This $\Pi$ should be regarded as an infinite sum of monomials in $x_{1}, x_{2}, \ldots$ and in $y_{1}, y_{2}, \ldots$ by expanding the factor corresponding to $i, j$ as a series at $x_{i} y_{j} \rightarrow 0$.

The property of $\Pi$ most useful for us can be stated as the following lemma. For any $f \in \Lambda$ denote by $f^{*}$ the operator on $\Lambda$ adjoint to the multiplication by $f$ relative to the bilinear form $(2.7)$. Note that here $f=f\left(x_{1}, x_{2}, \ldots\right)$. 
Lemma. We have

$$
f^{*}(\Pi) / \Pi=f\left(y_{1}, y_{2}, \ldots\right) \text {. }
$$

Proof. The commutative algebra $\Lambda$ is generated by the elements $p_{n}$ with $n \geqslant 1$. Therefore it suffices to prove (2.13) for $f=p_{n}$ only. Consider the operator $\partial / \partial p_{n}$ of derivation in $\Lambda$ relative to $p_{n}=p_{n}\left(x_{1}, x_{2}, \ldots\right)$. By the definition (2.7) we have

$$
p_{n}^{*}=\alpha n \partial / \partial p_{n}
$$

On the other hand, by taking the logarithm of (2.12) and then exponentiating,

$$
\Pi=\exp \left(\sum_{n=1}^{\infty} p_{n}\left(x_{1}, x_{2}, \ldots\right) p_{n}\left(y_{1}, y_{2}, \ldots\right) / \alpha n\right) \text {. }
$$

The relation (2.13) for $f=p_{n}$ follows from the last two displayed equalities.

2.5. Main result. Let $\mathbb{F}=\mathbb{Q}(\alpha)$ as in the previous two subsections. For $N \geqslant 1$ let $\rho_{N}$ be the homomorphism $\Lambda_{N} \rightarrow \Lambda_{N-1}$ defined by setting $x_{N}=0$, as in the beginning of Subsection 2.1. Denote

$$
A_{N}(u)=S_{N}(u) /(u)_{N}
$$

where we employ the Pochhammer symbol

$$
(u)_{N}=u(u+1) \ldots(u+N-1) .
$$

The right hand side of the equation (2.15) is regarded as a rational function of $u$ with the values being operators acting on the space $\Lambda_{N}$. Due to the stability property (2.10) of Jack symmetric polynomials, the equation (2.11) implies that

$$
\rho_{N} A_{N}(u)=A_{N-1}(u) \rho_{N}
$$

where $A_{0}(u)=1$. So the sequence of $A_{N}(u)$ with $N \geqslant 1$ has a limit at $N \rightarrow \infty$. This limit can be written as a series

$$
A(u)=1+A^{(1)} /(u)_{1}+A^{(2)} /(u)_{2}+\ldots
$$

where the inverses of the $(u)_{1},(u)_{2}, \ldots$ can be regarded as series in $u^{-1}$ whereas $A^{(1)}, A^{(2)}, \ldots$ are certain linear operators acting on $\Lambda$. By definition, the Jack symmetric functions are joint eigenvectors of these operators. In particular, the operators $A^{(1)}, A^{(2)}, \ldots$ pairwise commute, and are self-adjoint relative to the bilinear form (2.7). We call them the Sekiguchi-Debiard operators at infinity. Due to the property (2.10) their definition immediately implies that

$$
A^{(k)} P_{\lambda}=0 \quad \text { if } \quad \ell(\lambda)<k .
$$

It is also transparent from (2.11) that for any homogeneous $f \in \Lambda$

$$
A^{(1)} f=-\alpha \operatorname{deg} f .
$$

Hence in the notation (1.5)

$$
-A^{(1)}=H^{(1)} \text {. }
$$


The operator $A^{(2)}$ is well studied [Ex. VI.4.3]. In particular, it is known that

$$
A^{(1)}\left(A^{(1)}+1\right)-2 A^{(2)}=H^{(2)}
$$

in the notation (1.3). The main result of our article is the more general

Theorem. In the notation (2.2) for each $k=1,2, \ldots$ we have

$$
A^{(k)}=(-1)^{k} \sum_{\ell(\lambda)=k} d_{\lambda} m_{\lambda} m_{\lambda}^{*}
$$

where $\lambda$ ranges over all partitions of length $k$.

By inverting the relation (2.6) any monomial symmetric function $m_{\lambda}$ can be expressed as a linear combination of the functions $p_{\mu}$ where $\lambda, \mu$ are partitions of the same number and $\lambda \leqslant \mu$. By substituting into (2.18) and using (2.4),(2.14) one can write each operator $A^{(k)}$ in terms of $p_{n}$ and $\partial / \partial p_{n}$ where $n=1,2, \ldots$. In particular, one recovers the above formulas for the operators $A^{(1)}$ and $A^{(2)}$.

2.6. Step operators. In this subsection we will get a corollary to our theorem by using the following particular case of the Pieri rule for Jack symmetric functions. By [VI.6.24] for any partition $\mu$ the product $p_{1} P_{\mu}$ equals the linear combination of the symmetric functions $P_{\lambda}$ with the coefficients

$$
\prod_{j=1}^{i-1} \frac{\alpha\left(\lambda_{i}-\lambda_{j}\right)-i+j-1}{\alpha\left(\lambda_{i}-\lambda_{j}-1\right)-i+j} \cdot \prod_{j=1}^{i-1} \frac{\alpha\left(\lambda_{i}-\lambda_{j}-1\right)-i+j+1}{\alpha\left(\lambda_{i}-\lambda_{j}\right)-i+j}
$$

where $\lambda$ ranges over all partitions such that the sequence $\lambda_{1}, \lambda_{2}, \ldots$ is obtained from $\mu_{1}, \mu_{2}, \ldots$ by increasing one of its terms by 1 and $i$ is the index of the term.

Further, by [VI.6.19] the above stated equality implies that for any partition $\lambda$ the symmetric function $\partial P_{\lambda} / \partial p_{1}=\alpha^{-1} p_{1}^{*} P_{\lambda}$ equals the linear combination of the $P_{\mu}$ with the coefficients

$$
\prod_{j=1}^{\lambda_{i}-1} \frac{\alpha\left(\lambda_{i}-j-1\right)+\lambda_{j}^{\prime}-i+1}{\alpha\left(\lambda_{i}-j\right)+\lambda_{j}^{\prime}-i} \cdot \prod_{j=1}^{\lambda_{i}-1} \frac{\alpha\left(\lambda_{i}-j+1\right)+\lambda_{j}^{\prime}-i}{\alpha\left(\lambda_{i}-j\right)+\lambda_{j}^{\prime}-i+1}
$$

where $\mu$ ranges over all partitions such that the sequence $\mu_{1}, \mu_{2}, \ldots$ is obtained from $\lambda_{1}, \lambda_{2}, \ldots$ by decreasing one of its terms by 1 and $i$ is the index of the term. As usual, here $\lambda^{\prime}=\left(\lambda_{1}^{\prime}, \lambda_{2}^{\prime}, \ldots\right)$ is the partition conjugate to $\lambda$.

Now define the linear operators $B^{(1)}, B^{(2)}, \ldots$ acting on $\Lambda$ by setting

$$
B(u)=B^{(1)} /(u)_{1}+B^{(2)} /(u)_{2}+\ldots
$$

where

$$
\left[p_{1}, A(u)\right]=\alpha B(u)
$$

while the square brackets denote the operator commutator. Further, define the operators $C^{(1)}, C^{(2)}, \ldots$ acting on $\Lambda$ by setting

$$
C(u)=C^{(1)} /(u)_{1}+C^{(2)} /(u)_{2}+\ldots
$$


where

$$
\left[A(u), \partial / \partial p_{1}\right]=C(u) \text {. }
$$

Our definitions of the operators $B^{(1)}, B^{(2)}, \ldots$ and $C^{(1)}, C^{(2)}, \ldots$ are motivated by the results of [15]. Our theorem yields explicit expressions for these operators, stated as the following corollary. The corollary will then allow us to construct the elementary step operators for Jack symmetric functions, see (2.29) and (2.31).

Corollary. For every $k=0,1,2, \ldots$ we have the equalities

$$
\begin{aligned}
B^{(k+1)} & =(-1)^{k} \sum_{\ell(\mu)=k} d_{\mu \sqcup 1} m_{\mu \sqcup 1} m_{\mu}^{*}, \\
C^{(k+1)} & =(-1)^{k} \sum_{\ell(\mu)=k} d_{\mu \sqcup 1} m_{\mu} m_{\mu \sqcup 1}^{*}
\end{aligned}
$$

where $\mu \sqcup 1$ denotes the partition obtained from $\mu$ by appending one extra part 1 .

Proof. The equalities (2.23) and (2.24) follow from each other, because by (2.14)

$$
B(u)^{*}=C(u) .
$$

But the equality (2.24) follows from (2.18) and (2.22) by using [Ex. I.5.3].

By the definition (2.15) of the series $A(u)$, for any partition $\lambda$ we have

$$
A(u) P_{\lambda}=\prod_{i=1}^{\infty} \frac{u+i-1-\alpha \lambda_{i}}{u+i-1} \cdot P_{\lambda},
$$

see (2.11). In the infinite product displayed above the only factors different from 1 are those corresponding to $i=1, \ldots, \ell(\lambda)$. For any such $i$ consider the product

$$
\frac{1}{u+i-1} \prod_{\substack{j=1 \\ j \neq i}}^{\ell(\lambda)} \frac{u+j-1-\alpha \lambda_{j}}{u+j-1} .
$$

It follows from the definition (2.21) that for any given partition $\mu$ we have

$$
B(u) P_{\mu}=\sum_{\lambda} B_{\lambda \mu}(u) P_{\lambda}
$$

where $B_{\lambda \mu}(u)$ is the product of (2.19) by (2.26) while $\lambda$ ranges over all partitions such that the sequence $\lambda_{1}, \lambda_{2}, \ldots$ is obtained from $\mu_{1}, \mu_{2}, \ldots$ by increasing one of its terms by 1 and $i$ is the index of the term. Similarly, it follows from the definition (2.22) that for any given partition $\lambda$ we have

$$
C(u) P_{\lambda}=\sum_{\mu} C_{\mu \lambda}(u) P_{\mu}
$$

where $C_{\mu \lambda}(u)$ is the product of $(2.20)$ by $(2.26)$ and by $\alpha$ while $\mu$ ranges over all partitions such that the sequence $\mu_{1}, \mu_{2}, \ldots$ is obtained from $\lambda_{1}, \lambda_{2}, \ldots$ by decreasing one of its terms by 1 and $i$ is the index of the term. 
Let the partition $\lambda$ be fixed. Then for $i=1, \ldots, \ell(\lambda)$ the elements $\alpha \lambda_{i}-i+1$ of the field $\mathbb{Q}(\alpha)$ are pairwise distinct. Therefore by the part (i) of the corollary for the partition $\mu$ corresponding to any of these indices $i$ we have

$$
B\left(\alpha \lambda_{i}-i+1\right) P_{\mu}=B_{\lambda \mu}\left(\alpha \lambda_{i}-i+1\right) P_{\lambda}
$$

where the coefficient $B_{\lambda \mu}\left(\alpha \lambda_{i}-i+1\right)$ is the product of (2.19) by

$$
\prod_{j=1}^{\ell(\lambda)} \frac{1}{\alpha \lambda_{i}-i+j} \cdot \prod_{\substack{j=1 \\ j \neq i}}^{\ell(\lambda)}\left(\alpha \lambda_{i}-\alpha \lambda_{j}-i+j\right)
$$

The left hand side of the equality (2.29) should be understood as the value in $\Lambda$ of the rational function $B(u) P_{\mu}$ at the point $u=\alpha \lambda_{i}-i+1$. Similarly, by (ii)

$$
C\left(\alpha \lambda_{i}-i+1\right) P_{\lambda}=C_{\mu \lambda}\left(\alpha \lambda_{i}-i+1\right) P_{\mu}
$$

where $C_{\mu \lambda}\left(\alpha \lambda_{i}-i+1\right)$ is the product of $(2.20)$ by (2.30) and by $\alpha$.

2.7. Reduction of the proof. In this subsection we reduce the proof of our theorem to proving a certain determinantal identity for each $N=1,2, \ldots$. This identity will be proved in the next section by using the induction on $N$.

By the lemma from Subsection 2.4 our theorem is equivalent to the equality

$$
A(u)(\Pi) / \Pi=\sum_{k=0}^{\infty} \frac{(-1)^{k}}{(u)_{k}} \sum_{\ell(\lambda)=k} d_{\lambda} m_{\lambda}\left(x_{1}, x_{2}, \ldots\right) m_{\lambda}\left(y_{1}, y_{2}, \ldots\right)
$$

where the coefficients of the series $A(u)$ are regarded as operators acting on the symmetric functions in the variables $x_{1}, x_{2}, \ldots$. Here we set $(u)_{0}=1$. It suffices to prove for each $N=1,2, \ldots$ the restriction of the functional equality (2.32) to

$$
x_{N+1}=x_{N+2}=\ldots=0 .
$$

By the very definition of $A(u)$ the restriction of the left hand side of (2.32) to $(2.33)$ as of a function in the variables $x_{1}, x_{2}, \ldots$ equals

$$
A_{N}(u)\left(\Pi_{N}\right) / \Pi_{N}
$$

where we denote

$$
\Pi_{N}=\prod_{i=1}^{N} \prod_{j=1}^{\infty}\left(1-x_{i} y_{j}\right)^{-1 / \alpha}
$$

Simply by the definition of the monomial symmetric function $m_{\lambda}\left(x_{1}, x_{2}, \ldots\right)$ its restriction to $(2.33)$ is $m_{\lambda}\left(x_{1}, \ldots, x_{N}\right)$ if $\ell(\lambda) \leqslant N$ and vanishes if $\ell(\lambda)>N$. Therefore the restriction of the right hand side of (2.32) to (2.33) equals

$$
\sum_{k=0}^{N} \frac{(-1)^{k}}{(u)_{k}} \sum_{\ell(\lambda)=k} d_{\lambda} m_{\lambda}\left(x_{1}, \ldots, x_{N}\right) m_{\lambda}\left(y_{1}, y_{2}, \ldots\right) .
$$


Further, due to [VI.2.19] to prove the equality of (2.34) to (2.35) it suffices to set

$$
y_{N+1}=y_{N+2}=\ldots=0 .
$$

However, we will keep working with the infinite collection of variables $y_{1}, y_{2}, \ldots$. This will simplify the induction argument in the next section.

Let us compute the function (2.34). It depends on the variable $u$ rationally. It is also symmetric in either of the two collections of variables $x_{1}, \ldots, x_{N}$ and $y_{1}, y_{2}, \ldots$. This function can be obtained by applying to the identity function 1 the result of conjugating $A_{N}(u)$ by the operator of multiplication by $\Pi_{N}$.

Conjugating the operator (2.9) by $\Pi_{N}$ amounts to replacing each $\partial_{i}$ in $(2.9)$ with the sum

$$
\partial_{i}+\alpha^{-1} \sum_{l=1}^{\infty} \frac{y_{l}}{1-x_{i} y_{l}}
$$

Here we are just adding to each $\partial_{i}$ the logarithmic derivative of the function $\Pi_{N}$ relative to $x_{i}$. Hence conjugating $(2.9)$ by the multiplication by $\Pi_{N}$ yields

$$
\sum_{\sigma \in \mathfrak{S}_{N}}(-1)^{\sigma} \prod_{i=1}^{N}\left(x_{i}^{N-\sigma(i)}\left(u+\sigma(i)-1-\alpha x_{i} \partial_{i}-\sum_{l=1}^{\infty} \frac{x_{i} y_{l}}{1-x_{i} y_{l}}\right)\right) .
$$

Here in any single summand each of the factors corresponding to $i=1, \ldots, N$ does not depend on the variables $x_{j}$ with $j \neq i$. Therefore when applying the latter operator sum to the identity function 1 we can simply replace each $\partial_{i}$ with zero. Then we get the function

$$
\begin{gathered}
\sum_{\sigma \in \mathfrak{S}_{N}}(-1)^{\sigma} \prod_{i=1}^{N}\left(x_{i}^{N-\sigma(i)}\left(u+\sigma(i)-1-\sum_{l=1}^{\infty} \frac{x_{i} y_{l}}{1-x_{i} y_{l}}\right)\right)= \\
\operatorname{det}\left[x_{i}^{N-j}\left(u+j-1+\sum_{l=1}^{\infty} \frac{x_{i} y_{l}}{x_{i} y_{l}-1}\right)\right]_{i, j=1}^{N} .
\end{gathered}
$$

It follows that the function (2.34) is equal to the determinant (2.36) divided by the Vandermonde polynomial $\Delta\left(x_{1}, \ldots, x_{N}\right)$ and by the Pochhammer symbol $(u)_{N}$, see (2.8) and (2.15). This ratio is equal to the right hand side of (2.35) by the proposition in Subsection 3.1, see the argument at the end of that subsection. We will prove the proposition in Subsections 3.2 to 3.4. Thus we will complete the proof of our theorem. Note that another proof of this theorem can be obtained by using the results of [2, Sec. 3] and [17, Sec. 9] on the Macdonald operators.

\section{Determinantal identities}

3.1. Getting the theorem. Let $\mathbb{F}$ be any field. Consider the rational function of two variables $u, v$

$$
\Psi(u, v)=\frac{u v}{u v-1}
$$

with values in $\mathbb{F}$. This function is a solution of the equation

$$
(u-v) \Psi(u, w) \Psi(v, w)=u \Psi(v, w)-v \Psi(u, w) .
$$


Here $w$ is a third variable. One can easily demonstrate that any non-zero rational solution $\Psi(u, v)$ of $(3.2)$ has the form $u /(u-\psi(v))$ where $\psi(v)$ is an arbitrary rational function of a single variable. In particular, by choosing $\psi(v)=1 / v$ we get the solution (3.1).

Let $x_{1}, \ldots, x_{N}$ and $y_{1}, y_{2}, \ldots$ be independent variables. Here we assume that $N \geqslant 1$. In the next three subsections we will prove the following proposition.

Proposition. For any solution $\Psi(u, v)$ of the equation (3.2) we have an identity

$$
\begin{gathered}
\left.\operatorname{det}\left[x_{i}^{N-j}\left(u+j-1+\sum_{l=1}^{\infty} \Psi\left(x_{i}, y_{l}\right)\right)\right]^{N}\right]_{i, j=1}^{N}= \\
\Delta\left(x_{1}, \ldots, x_{N}\right) \sum_{k=0}^{N}(u+k) \ldots(u+N-1) \sum_{\substack{i_{1}, \ldots, i_{k} \\
j_{1}, \ldots, j_{k}}} \prod_{r=1}^{k} \Psi\left(x_{i_{r}}, y_{j_{r}}\right)
\end{gathered}
$$

where all the indices $i_{1}, \ldots, i_{k} \in\{1, \ldots, N\}$ are distinct, the indices $j_{1}, \ldots, j_{k} \in$ $\{1,2, \ldots\}$ are all distinct too, and the sum is taken over all collections of these indices such that different are all the corresponding sets of $k$ pairs

$$
\left\{\left(i_{1}, j_{1}\right), \ldots,\left(i_{k}, j_{k}\right)\right\} \text {. }
$$

For any $k \geqslant 1$ take the symmetric group $\mathfrak{S}_{k}$. Using the permutations $\sigma \in \mathfrak{S}_{k}$ the sum over the indices $i_{1}, \ldots, i_{k}$ and $j_{1}, \ldots, j_{k}$ at the right hand side of the equality (3.3) can be also written as

$$
\sum_{\substack{i_{1}<\ldots<i_{k} \\ j_{1}<\ldots<j_{k}}} \sum_{\sigma \in \mathfrak{S}_{k}} \prod_{r=1}^{k} \Psi\left(x_{i_{r}}, y_{j_{\sigma(r)}}\right) .
$$

Hence by choosing the function $\Psi(u, v)$ as in (3.1) our proposition implies that the determinant (2.36) equals

$$
\begin{gathered}
\Delta\left(x_{1}, \ldots, x_{N}\right) \sum_{k=0}^{N}(u+k) \ldots(u+N-1) \times \\
\sum_{\ell(\lambda)=k} d_{\lambda} m_{\lambda}\left(x_{1}, \ldots, x_{N}\right) m_{\lambda}\left(y_{1}, y_{2}, \ldots\right) .
\end{gathered}
$$

Indeed, if $\Psi(u, v)$ is the rational function (3.1) then the sum (3.5) equals

$$
\begin{gathered}
\sum_{\substack{i_{1}<\ldots<i_{k} \\
j_{1}<\ldots<j_{k}}} \sum_{\sigma \in \mathfrak{S}_{k}} \prod_{r=1}^{k} \frac{x_{i_{r}} y_{j_{\sigma(r)}}}{x_{i_{r}} y_{j_{\sigma(r)}}-1}= \\
\sum_{\substack{i_{1}<\ldots<i_{k} \\
j_{1}<\ldots<j_{k}}} \sum_{\sigma \in \mathfrak{S}_{k}} \sum_{l_{1}, \ldots, l_{k}=1}^{\infty} \prod_{r=1}^{k}\left(x_{i_{r}} y_{j_{\sigma(r)}}\right)^{l_{r}}= \\
\sum_{\substack{i_{1}<\ldots<i_{k} \\
j_{1}<\ldots<j_{k}}} \sum_{\sigma, \tau \in \mathfrak{S}_{k}} \sum_{\ell(\lambda)=k} d_{\lambda}^{-1} \prod_{r=1}^{k}\left(x_{i_{r}} y_{j_{\sigma(r)}}\right)^{\lambda_{\tau(r)}} .
\end{gathered}
$$


Here $\lambda$ ranges over all partitions $\lambda$ of length $k$. The sum displayed in the last line equals the sum in the second line of (3.6), by using the expression (2.1) for the polynomial $m_{\lambda}\left(x_{1}, \ldots, x_{N}\right)$ and a similar expression for $m_{\lambda}\left(y_{1}, y_{2}, \ldots\right)$. Thus our proposition implies the theorem as stated in Subsection 2.5.

3.2. Expanding the determinant. We will prove the proposition by induction on $N$. The case $N=1$ is the induction base. Here the left hand side of (3.3) is

$$
u+\sum_{l=1}^{\infty} \Psi\left(x_{1}, y_{l}\right)
$$

The right hand side of (3.3) is then the same by definition, since $\Delta\left(x_{1}\right)=1$.

Now take $N>1$ and assume that the identity (3.3) holds for $N-1$ instead of $N$. For each index $i=1, \ldots, N$ we will for short denote

$$
\Delta_{i}=\Delta\left(x_{1}, \ldots, \widehat{x}_{i}, \ldots, x_{N}\right)
$$

where as usual the symbol $\widehat{x}_{i}$ indicates the omitted variable. By expanding the determinant at the left hand side of (3.3) in the first column and then using the induction assumption with $u+1$ instead of $u$, we get the sum

$$
\begin{gathered}
\sum_{i=1}^{N}(-1)^{i+1} x_{i}^{N-1} u \Delta_{i} \times \\
\sum_{k=0}^{N-1}(u+k+1) \ldots(u+N-1) \sum_{\substack{i_{1}, \ldots, i_{k} \neq i \\
j_{1}, \ldots, j_{k}}} \prod_{r=1}^{k} \Psi\left(x_{i_{r}}, y_{j_{r}}\right)+ \\
\sum_{i=1}^{N}(-1)^{i+1} x_{i}^{N-1} \sum_{l=1}^{\infty} \Psi\left(x_{i}, y_{l}\right) \Delta_{i} \times \\
\sum_{k=0}^{N-1}(u+k+1) \ldots(u+N-1) \sum_{\substack{i_{1}, \ldots, i_{k} \neq i \\
j_{1}, \ldots, j_{k}}} \prod_{r=1}^{k} \Psi\left(x_{i_{r}}, y_{j_{r}}\right) .
\end{gathered}
$$

Now consider the sum

$$
\sum_{i=1}^{N}(-1)^{i+1} x_{i}^{N-1} \Delta_{i} \sum_{l=1}^{\infty} \sum_{\substack{i_{1}, \ldots, i_{k} \neq i \\ j_{1}, \ldots, j_{k}}} \Psi\left(x_{i}, y_{l}\right) \prod_{r=1}^{k} \Psi\left(x_{i_{r}}, y_{j_{r}}\right)
$$

coming from the last two lines of the display (3.7). This sum can be written as

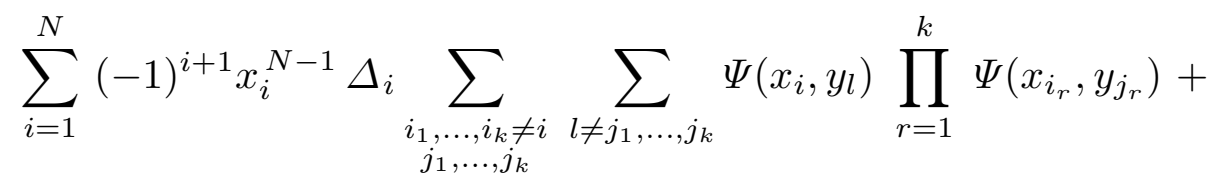

$$
\begin{aligned}
& \sum_{i=1}^{N}(-1)^{i+1} x_{i}^{N-1} \Delta_{i} \sum_{\substack{i_{1}, \ldots, i_{k} \neq i \\
j_{1}, \ldots, j_{k}}} \sum_{s=1}^{k} \Psi\left(x_{i}, y_{j_{s}}\right) \prod_{r=1}^{k} \Psi\left(x_{i_{r}}, y_{j_{r}}\right)
\end{aligned}
$$


The next two subsections will show that the sum in the second line of (3.8) equals

$$
k \sum_{i=1}^{N}(-1)^{i+1} x_{i}^{N-1} \Delta_{i} \sum_{\substack{i_{1}, \ldots, i_{k} \neq i \\ j_{1}, \ldots, j_{k}}} \prod_{r=1}^{k} \Psi\left(x_{i_{r}}, y_{j_{r}}\right) .
$$

Due to that equality the sum (3.7) can be rewritten as

$$
\begin{gathered}
\sum_{i=1}^{N}(-1)^{i+1} x_{i}^{N-1} \Delta_{i} \times \\
\left(\sum_{k=0}^{N-1}(u+k)(u+k+1) \ldots(u+N-1) \sum_{\substack{i_{1}, \ldots, i_{k} \neq i \\
j_{1}, \ldots, j_{k}}} \prod_{r=1}^{k} \Psi\left(x_{i_{r}}, y_{j_{r}}\right)+\right. \\
\left.\sum_{k=0}^{N-1}(u+k+1) \ldots(u+N-1) \sum_{\substack{i_{1}, \ldots, i_{k} \neq i \\
j_{1}, \ldots, j_{k} \neq l}}^{k} \Psi\left(x_{i}, y_{l}\right) \prod_{r=1}^{k} \Psi\left(x_{i_{r}}, y_{j_{r}}\right)\right)
\end{gathered}
$$

Here the indices $i_{1}, j_{1}, \ldots, i_{k}, j_{k}$ and $l$ are assumed to be running. In the second line of (3.10) we can include the index $k=N$ to the summation range without affecting the sum since $k$ distinct indices $i_{1}, \ldots, i_{k} \neq i$ exist only if $k<N$. In the third line we can replace $k+1$ with $k$ where $k=1, \ldots, N$. We get

$$
\sum_{k=1}^{N}(u+k) \ldots(u+N-1) \sum_{\substack{i_{1}, \ldots, i_{k-1}, i \\ j_{1}, \ldots, j_{k-1}, l}} \Psi\left(x_{i}, y_{l}\right) \prod_{r=1}^{k-1} \Psi\left(x_{i_{r}}, y_{j_{r}}\right)
$$

Here the set

$$
\left\{\left(\left(i_{1}, j_{1}\right), \ldots,\left(i_{k-1}, j_{k-1}\right),(i, l)\right\}\right.
$$

can be any of the sets (3.4) appearing in (3.3), provided that in (3.4) one of the indices $i_{1}, \ldots, i_{k}$ coincides with the given index $i$. That one index can be then denoted by $i_{k}$ because the order of the $k$ elements of the set (3.4) does not matter.

These observations will show that the sum displayed in the second and the third lines of of (3.10) equals

$$
\sum_{k=0}^{N}(u+k) \ldots(u+N-1) \sum_{\substack{i_{1}, \ldots, i_{k} \\ j_{1}, \ldots, j_{k}}} \prod_{r=1}^{k} \Psi\left(x_{i_{r}}, y_{j_{r}}\right)
$$

In particular, they will show that this sum does not depend on the index $i$. Hence we will have the identity (3.3) proved, by expanding the determinant $\Delta\left(x_{1}, \ldots, x_{N}\right)$ in the first column. 
3.3. Two sums. We need to prove for $k=0, \ldots, N-1$ that the sum (3.9) equals the sum in the second line of (3.8). By using (3.2) the last mentioned sum can be written as

$$
\begin{gathered}
\sum_{i=1}^{N} \sum_{\substack{i_{1}, \ldots, i_{k} \neq i \\
j_{1}, \ldots, j_{k}}} \sum_{s=1}^{k}(-1)^{i+1} x_{i}^{N-1} \Delta_{i} \times \\
\frac{x_{i} \Psi\left(x_{i_{s}}, y_{j_{s}}\right)-x_{i_{s}} \Psi\left(x_{i}, y_{j_{s}}\right)}{x_{i}-x_{i_{s}}} \prod_{r \neq s} \Psi\left(x_{i_{r}}, y_{j_{r}}\right)
\end{gathered}
$$

which by subtracting the product $x_{i_{s}} \Psi\left(x_{i_{s}}, y_{j_{s}}\right)$ from the numerator of the above displayed fraction and then adding it back can be rewritten as

$$
\begin{gathered}
\sum_{i=1}^{N} \sum_{\substack{i_{1}, \ldots, i_{k} \neq i \\
j_{1}, \ldots, j_{k}}} \sum_{s=1}^{k}(-1)^{i+1} x_{i}^{N-1} \Delta_{i} \prod_{r=1}^{k} \Psi\left(x_{i_{r}}, y_{j_{r}}\right)+ \\
\sum_{i=1}^{N} \sum_{\substack{i_{1}, \ldots, i_{k} \neq i \\
j_{1}, \ldots, j_{k}}} \sum_{s=1}^{k}(-1)^{i+1} x_{i}^{N-1} \Delta_{i} \times \\
\left(\frac{x_{i_{s}} \Psi\left(x_{i_{s}}, y_{j_{s}}\right)}{x_{i}-x_{i_{s}}}+\frac{x_{i_{s}} \Psi\left(x_{i}, y_{j_{s}}\right)}{x_{i_{s}}-x_{i}}\right) \prod_{r \neq s} \Psi\left(x_{i_{r}}, y_{j_{r}}\right) .
\end{gathered}
$$

The summands in the first line of the display (3.11) do not depend on $s$. Hence their sum equals (3.9). Let us show that the sum appearing in the second and the third lines of (3.11) equals zero. By opening the brackets in the third line and then swapping the running indices $i, i_{s}$ in each term coming from the second fraction in the brackets, the sum in the second and the third lines becomes

$$
\begin{gathered}
\sum_{i=1}^{N} \sum_{\substack{i_{1}, \ldots, i_{k} \neq i \\
j_{1}, \ldots, j_{k}}} \sum_{s=1}^{k}\left(\prod_{r=1}^{k} \Psi\left(x_{i_{r}}, y_{j_{r}}\right)\right) \times \\
\frac{(-1)^{i+1} x_{i}^{N-1} x_{i_{s}} \Delta_{i}+(-1)^{i_{s}+1} x_{i_{s}}^{N-1} x_{i} \Delta_{i_{s}}}{x_{i}-x_{i_{s}}}
\end{gathered}
$$

Here in the first line the product over $r=1, \ldots, k$ depends neither on the index $s$ nor on the choice of the index $i \neq i_{1}, \ldots, i_{k}$. The fraction in the second line does not depend on the indices $j_{1}, \ldots, j_{k}$. We will show that for any fixed distinct indices $i_{1}, \ldots, i_{k} \in\{1, \ldots, N\}$ the sum of these fractions over $s=1, \ldots, k$ and $i \neq i_{1}, \ldots, i_{k}$ is equal to zero. This will complete our proof of the identity (3.3).

Let $\sigma$ range over $\mathfrak{S}_{N}$. By the definition of the Vandermonde polynomial, the sum of the last displayed fractions over $s=1, \ldots, k$ and $i \neq i_{1}, \ldots, i_{k}$ equals

$$
\sum_{i \neq i_{1}, \ldots, i_{k}} \sum_{s=1}^{k} \sum_{\sigma(i)=1}(-1)^{\sigma} \frac{x_{i}^{N-1} x_{i_{s}}^{N-\sigma\left(i_{s}\right)+1}-x_{i_{s}}^{N-1} x_{i}^{N-\sigma\left(i_{s}\right)+1}}{x_{i}-x_{i_{s}}} \prod_{j \neq i, i_{s}} x_{j}^{N-\sigma(j)}
$$


which is in turn equal to the sum

$$
\sum_{i \neq i_{1}, \ldots, i_{k}} \sum_{s=1}^{k} \sum_{\sigma(i)=1}(-1)^{\sigma} \sum_{r=2}^{\sigma\left(i_{s}\right)-1} x_{i}^{N-r} x_{i_{s}}^{N-\sigma\left(i_{s}\right)+r-1} \prod_{j \neq i, i_{s}} x_{j}^{N-\sigma(j)} .
$$

Recall that here $N>1$. One can easily prove by induction on $N=2,3, \ldots$ that the total number of terms in the sum (3.12) equals

$$
(N-1) !(N-2)(N-k) k / 2 .
$$

However, we shall not use this equality and will leave its proof to the reader. In the next subsection, we will prove that all terms in (3.12) cancel each other.

3.4. Cancellations. The sum (3.12) is taken over quadruples $(i, s, \sigma, r)$. Take any of them such that

$$
\sigma^{-1}(r) \neq i_{1}, \ldots, i_{k}
$$

Such a quadruple will be called of type $I$. Denote $\sigma^{-1}(r)=\bar{\imath}$. Observe that $\bar{\imath} \neq i$ because $\sigma(\bar{\imath})=r \geqslant 2$ while $\sigma(i)=1$. Put

$$
\bar{\sigma}=\sigma \tau_{i \bar{\imath}}
$$

where $\tau_{i \bar{\imath}} \in \mathfrak{S}_{N}$ is the transposition of $i$ and $\bar{\imath}$. Then $\bar{\sigma}(i)=r$ and $\bar{\sigma}(\bar{\imath})=1$. We also have $\bar{\sigma}\left(i_{s}\right)=\sigma\left(i_{s}\right)$ because $i_{s} \neq i, \bar{\imath}$. Hence the quadruple $(\bar{\imath}, s, \bar{\sigma}, r)$ appears in (3.12) together with the quadruple $(i, s, \sigma, r)$. But the summands in (3.12) corresponding to these two quadruples cancel each other. Indeed,

$$
\begin{gathered}
-(-1)^{\sigma} x_{i}^{N-r} x_{\bar{\imath}}^{N-\sigma(\bar{\imath})} x_{i_{s}}^{N-\sigma\left(i_{s}\right)+r-1} \prod_{j \neq i, \bar{\imath}, i_{s}} x_{j}^{N-\sigma(j)}= \\
(-1)^{\bar{\sigma}} x_{i}^{N-\bar{\sigma}(i)} x_{\bar{\imath}}^{N-r} x_{i_{s}}^{N-\bar{\sigma}\left(i_{s}\right)+r-1} \prod_{j \neq i, \bar{\imath}, i_{s}} x_{j}^{N-\bar{\sigma}(j)} .
\end{gathered}
$$

Note that here

$$
\bar{\sigma}^{-1}(r)=i \neq i_{1}, \ldots, i_{k}
$$

hence $(\bar{\imath}, s, \bar{\sigma}, r)$ is also of type I. Moreover, by applying our construction to the latter quadruple instead of $(i, s, \sigma, r)$ we get the initial quadruple $(i, s, \sigma, r)$ back.

Next take a quadruple $(i, s, \sigma, r)$ showing in (3.12) such that for some index $\bar{s}$

$$
\sigma\left(i_{s}\right)-r+1=\sigma\left(i_{\bar{s}}\right) .
$$

Such a quadruple will be called of type $I I$. Note that $s \neq \bar{s}$ because $r \neq 1$. Put

$$
\bar{\sigma}=\sigma \tau_{i_{s} i_{\bar{s}}} .
$$

Here $\bar{\sigma}(i)=\sigma(i)=1$ because $i \neq i_{s}, i_{\bar{s}}$. We also have $\bar{\sigma}\left(i_{\bar{s}}\right)=\sigma\left(i_{s}\right)$. Hence the quadruple $(i, \bar{s}, \bar{\sigma}, r)$ appears in (3.12) together with $(i, s, \sigma, r)$. The summands in (3.12) corresponding to these two quadruples cancel each other. Indeed, 


$$
\begin{aligned}
& -(-1)^{\sigma} x_{i}^{N-r} x_{i_{s}}^{N-\sigma\left(i_{s}\right)+r-1} x_{i_{\bar{s}}}^{N-\sigma\left(i_{\bar{s}}\right)} \prod_{j \neq i, i_{s}, i_{\bar{s}}} x_{j}^{N-\sigma(j)}= \\
& (-1)^{\bar{\sigma}} x_{i}^{N-r} x_{i_{s}}^{N-\bar{\sigma}\left(i_{s}\right)} x_{i_{\bar{s}}}^{N-\bar{\sigma}\left(i_{\bar{s}}\right)+r-1} \prod_{j \neq i, i_{s}, i_{\bar{s}}} x_{j}^{N-\bar{\sigma}(j)} .
\end{aligned}
$$

Here

$$
\bar{\sigma}\left(i_{\bar{s}}\right)-r+1=\sigma\left(i_{s}\right)-r+1=\bar{\sigma}\left(i_{s}\right)
$$

and $(i, \bar{s}, \bar{\sigma}, r)$ is also of type II. Moreover, by applying our construction to the latter quadruple instead of $(i, s, \sigma, r)$ we get the initial quadruple $(i, s, \sigma, r)$ back.

Note that the above two constructions differ for the quadruples of type I and II, while a quadruple can be of both types simultaneously. However, the summands in (3.12) corresponding to quadruples of any of the two types still cancel each other. Indeed, take any quadruple $(i, s, \sigma, r)$ of type I which also has type II. By applying our first constuction to it we get another quadruple $(\bar{\imath}, s, \bar{\sigma}, r)$ of type I, where $\bar{\sigma}$ is defined by (3.13) while $\bar{\imath}=\sigma^{-1}(r)$. But then for a certain index $\bar{s}$ we have

$$
\bar{\sigma}\left(i_{s}\right)-r+1=\sigma\left(i_{s}\right)-r+1=\sigma\left(i_{\bar{s}}\right)
$$

because $(i, s, \sigma, r)$ also has type II. Hence the quadruple $(\bar{\imath}, s, \bar{\sigma}, r)$ is of type II too. We could similarly check that the result of applying our second construction to $(i, s, \sigma, r)$ is not only of type II but of type I as well. However, this is already not needed for the cancellation. So we will leave checking it to the reader.

Finally, take any quadruple $(i, s, \sigma, r)$ appearing in (3.12) which is neither of type I nor of type II. Such a quadruple will be called of type III. Here $r=\sigma\left(i_{\bar{s}}\right)$ for some index $\bar{s}$ because $(i, s, \sigma, r)$ is not of type I . For some index $\bar{\imath} \neq i_{1}, \ldots, i_{k}$ we also have

$$
\sigma\left(i_{s}\right)-r+1=\sigma(\bar{\imath})
$$

because $(i, s, \sigma, r)$ is not of type II . Observe that here the four indices $i, i_{\bar{s}}, i_{s}, \bar{\imath}$ are pairwise distinct. Indeed, here we have $i, \bar{\imath} \neq i_{s}, i_{\bar{s}}$ by definition. Further, here $i \neq \bar{\imath}$ because $\sigma(i)=1$ while $\sigma(\bar{\imath}) \geqslant 2$ by the definition (3.14). Furthermore, here $i_{s} \neq i_{\bar{s}}$ because $r<\sigma\left(i_{s}\right)$ while $r=\sigma\left(i_{\bar{s}}\right)$. Put $\bar{\sigma}=\sigma \tau$ where $\tau \in \mathfrak{S}_{N}$ cyclically permutes the indices $i, i_{\bar{s}}, i_{s}, \bar{\imath}$ and leaves all the remaining indices fixed. More exactly,

$$
\tau: i \mapsto i_{\bar{s}} \mapsto i_{s} \mapsto \bar{\imath} \mapsto i
$$

Let $\bar{r}$ be the number at either side of equality (3.14). Consider the quadruple $(\bar{\imath}, \bar{s}, \bar{\sigma}, \bar{r})$. Here $\bar{\sigma}(\bar{\imath})=\sigma(i)=1$ by definition. Due to the range of $r$ we also have

$$
2 \leqslant \bar{r} \leqslant \sigma\left(i_{s}\right)-1=\bar{\sigma}\left(i_{\bar{s}}\right)-1 .
$$

Therefore the quadruple $(\bar{\imath}, \bar{s}, \bar{\sigma}, \bar{r})$ appears in (3.12) together with $(i, s, \sigma, r)$. The summands in (3.12) corresponding to the two quadruples cancel each other. Indeed, we have the equality 


$$
\begin{aligned}
& -(-1)^{\sigma} x_{i}^{N-r} x_{i_{\bar{s}}}^{N-\sigma\left(i_{\bar{s}}\right)} x_{i_{s}}^{N-\sigma\left(i_{s}\right)+r-1} x_{\bar{\imath}}^{N-\sigma(\bar{\imath})} \prod_{j \neq i, i_{\bar{s}}, i_{s}, \bar{\imath}} x_{j}^{N-\sigma(j)}=
\end{aligned}
$$

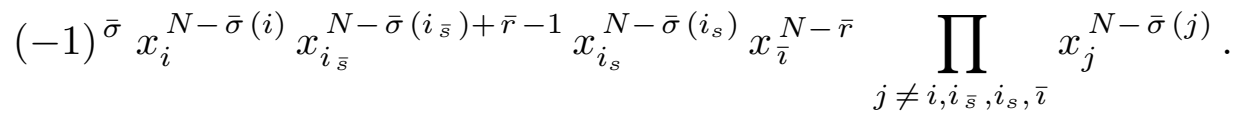

Here $\bar{r}=\bar{\sigma}\left(i_{s}\right)$ so that $(\bar{\imath}, \bar{s}, \bar{\sigma}, \bar{r})$ is not of type I. We also have

$$
\bar{\sigma}\left(i_{\bar{s}}\right)-\bar{r}+1=\bar{\sigma}(i)
$$

so that $(\bar{\imath}, \bar{s}, \bar{\sigma}, \bar{r})$ is not of type II . So this quadruple is of type III. Moreover, by applying our third construction to this quadruple instead of $(i, s, \sigma, r)$ we get the initial quadruple $(i, s, \sigma, r)$ back. Thus all summands in (3.12) cancel each other. We have now completed the induction step in the proof of our proposition.

\section{Acknowledgements}

This work originates from our discussions with S. M. Khoroshkin. It was he who suggested to us to compute limits of commuting operators whose eigenvectors are the Jack symmetric polynomials. We are also grateful to A. K. Pogrebkov for helpful comments on his work [12], and to E. Vasserot for drawing our attention to his joint work with O. Schiffmann [15]. The first and second named of us have been supported by the EPSRC grants EP/I014071 and EP/H000054 respectively.

\section{References}

.1 A. G. Abanov and P.B. Wiegmann, Quantum Hydrodynamics, the quantum BenjaminOno equation, and the Calogero model, Phys. Rev. Lett. 95 (2005), 076402.

.2 H. Awata and H. Kanno, Macdonald operators and homological invariants of the colored Hopf link, J. Phys. A 44 (2011), 375201.

.3 H. Awata, Y. Matsuo, S. Odake and J.Shiraishi, Collective fields, Calogero-Sutherland model and generalized matrix models, Phys. Lett. B 347 (1995), 49-55.

.4 W. Cai and N. Jing, Applications of Laplace-Beltrami operator for Jack polynomials, European J. Combin. 33 (2012), 556-571.

$.5 \quad$ F. Calogero, Ground state of a one-dimensional N-body system, J. Math. Phys. 10 (1969), 2197-2200.

.6 A. Debiard, Polynômes de Tchébychev et de Jacobi dans un espace euclidien de dimension $p$, C. R. Acad. Sc. Paris I 296 (1983), 529-532.

.7 L. Lapointe and L. Vinet, Exact operator solution of the Calogero-Sutherland model, Comm. Math. Phys. 178 (1996), 425-152.

.8 I. G. Macdonald, Symmetric Functions and Hall Polynomials, Oxford University Press, 1995.

.9 J. A. Minahan and A. P. Polychronakos, Density correlation functions in Calogero-Sutherland models, Phys. Rev. B 50 (1994), 4236-4239.

.10 M. L. Nazarov and E. K. Sklyanin, Macdonald operators at infinity, arXiv:1212.2960

.11 A. Okounkov and R. Pandharipande, Quantum cohomology of the Hilbert scheme of points in the plane, Inv. Math. 179 (2010), 523-557.

.12 A. K. Pogrebkov, Boson-fermion correspondence and quantum integrable and dispersionless models, Russ. Math. Surv. 58 (2003), 1003-1037.

.13 A. P. Polychronakos, Waves and solitons in the continuum limit of the Calogero-Sutherland model, Phys. Rev. Lett. 74 (1995), 5153-5157.

.14 P. Rossi, Gromov-Witten invariants of target curves via Symplectic Field Theory, J. Geom. Phys. 58 (2008), 931-941. 
.15 O. Schiffmann and E. Vasserot, Cherednik algebras, W-algebras and the equivariant cohomology of the moduli space of instantons on $\mathbb{A}^{2}$, arXiv:1202.2756

.16 J. Sekiguchi, Zonal spherical functions on some symmetric spaces, Publ. Res. Inst. Math. Sci. 12 (1977), 455-459.

.17 J. Shiraishi, A family of integral transformations and basic hypergeometric series, Comm. Math. Phys. 263 (2006), 439-460.

.18 E. K.Sklyanin, Separation of variables. New trends, Progress Theor. Phys. Suppl. 118 (1995), 35-60.

.19 B. Sutherland, Exact results for a quantum many-body problem in one dimension, Phys. Rev. A 4 (1971), 2019-2021.

.20 B. Sutherland, Exact results for a quantum many-body problem in one dimension II, Phys. Rev. A 5 (1972), 1372-1376. 